\title{
Comprehensive analysis of miRNA-mRNA-IncRNA networks in severe asthma
}

\author{
Yi Chen ${ }^{\ddagger}{ }^{1}$, Zheng-Dao Mao ${ }^{\ddagger}{ }^{1}$, Yu-Jia Shi ${ }^{1}$, Yan Qian ${ }^{1}$, Zhi-Guang Liu ${ }^{1}$, Xiao-Wei Yin ${ }^{1}$ \& \\ Qian Zhang*,1 \\ ${ }^{1}$ Department of Respiratory and Critical Care Medicine, The Affiliated Changzhou No.2 People's Hospital of Nanjing Medical \\ University, 29 Xinlong Road, Changzhou 213003, PR China \\ *Author for correspondence: Tel.: +86 05198108 7391; Fax: +86 05198811 5560; zhang_qiannj@163.com \\ ${ }^{\ddagger}$ Authors contributed equally
}

\begin{abstract}
Aim: This study aimed to explore the molecular mechanism of severe asthma. Materials \& methods: The shared and divergent differentially expressed mRNAs (DEmRNAs), miRNAs (DEmiRNAs) and IncRNAs (DElncRNAs) in asthma and severe asthma were identified by RNA-sequencing. Severe asthmaspecific and shared DEmiRNA-DEmRNA-DEIncRNA interaction networks were performed. Results: Compared with normal control, 1328 DEmRNAs, 608 DElncRNAs and 63 DEmiRNAs were identified in severe asthma. Compared with asthma, 95 DEmRNAs, 143 DEIncRNAs and 96 DEmiRNAs were identified in severe asthma. MiR-133a-3p-EFHD2/CNN2-AC144831.1 interactions and miR-3613-3p-CD44/BCL11BLINC00158/CTA-217C2.1/AC010976.2/RP11-641A6.2 interactions were speculated to involve with the development of severe asthma. The results of GSE69683 validation were generally consistent with our RNAsequencing results. Conclusion: This study provides clues for understanding the mechanism of severe asthma.
\end{abstract}

First draft submitted: 17 August 2018; Accepted for publication: 25 October 2018; Published online: 14 November 2018

Keywords: asthma $\bullet$ comprehensive analysis $\bullet$ DEmiRNA-DEmRNA-DElncRNA interaction networks $\bullet$ IncRNA $\bullet$ miR133A-3P • miR-361-3p • miRNA • mRNA • RNA-sequencing • severe asthma

Asthma is a common chronic disease of the airways that affects 300 million people worldwide, with that number expected to rise [1]. Asthma is characterized by airway inflammation, smooth muscle bronchoconstriction leading to airflow obstruction, and mucous hypersecretion [2]. Severe asthma is a subset of difficult-to-treat asthma, that requires treatment with high dose inhaled corticosteroids plus a second controller and/or systemic corticosteroids to prevent it from becoming 'uncontrolled' or that 'uncontrolled' despite this therapy [3]. Patients with severe asthma accounts for 3-10\% [3,4] of the population of adults with asthma, but leads to increased morbidity and their care is estimated to make up more an $60 \%$ of the costs associated with asthma [5-9]. However, the pathology of severe asthma remains elusive. It is urgent to explore the pathology and develop biomarkers for severe asthma.

Non-coding RNAs (ncRNAs) are functional RNAs which have no ability of coding proteins. MiRNAs are a class of small ncRNAs $(\approx 22 \mathrm{nts})$ which play multiple roles in various biological processes by regulating expression of their target genes [10]. Long noncoding cRNAs (lncRNAs) is a type of ncRNAs with a length of longer than 200 nts which are emerging as key regulators in various biological processes, including chromatin modification, transcription and post-transcriptional processing [11]. Accumulated evidences indicated that the interactions between lncRNAs and miRNAs, and their downstream target genes have been closely related to various diseases.

In this study, we obtained the mRNA, miRNA and lncRNA expression data from, asthma, severe asthma and normal controls by using RNA-sequencing. Moreover, miRNA-mRNA-lncRNA networks were constructed to elaborate the interactions and potential cross talk among the key lncRNAs, miRNAs and mRNAs in asthma and severe asthma. 
Table 1. Patient characteristics.

\begin{tabular}{|llll|}
\hline Indicators & Normal control & Asthma & Severe asthma \\
\hline Mean age (years) & $51.67 \pm 12.01$ & $52.33 \pm 15.31$ & $53.66 \pm 11.68$ \\
\hline Mean BMI & $21.31 \pm 1.30$ & $21.17 \pm 1.72$ & $20.73 \pm 5.53$ \\
\hline Gender (male/female) & $1 / 2$ & $1 / 2$ & $1 / 2$ \\
\hline FEV1\% & $125.2 \pm 1.51$ & $73.83 \pm 15.54$ & $41.53 \pm 16.84$ \\
\hline FEV1/FVC & $87.63 \pm 10.37$ & $76.2 \pm 17.94$ & $52.10 \pm 23.56$ \\
\hline FEV1\%: Percent of predicted forced expiratory volume in 1 second; FEV1/FVC: FEV1 as percentage of forced vital capacity.
\end{tabular}

\section{Materials \& methods}

\section{Patients \& samples}

Three adult patients with asthma, three with severe asthma and three control subjects enrolled in our study were from the Affiliated Changzhou No.2 People's Hospital of Nanjing Medical University. The inclusion criteria were as follows: patients with asthma were diagnosed based on Bronchial Asthma Prevention Guidelines of Chinese Medicine Academy [12]; patients with severe asthma were diagnosed based on the expert consensus on the diagnosis and treatment of severe asthma [13]; participants more than 18; and participants did not take any drugs during the short period of sample collection time. Participants with abnormal liver function, coinfection, pulmonary embolism, chronic bronchitis, tuberculosis and blood system diseases were excluded. The detailed information of all participants were displayed in Table 1. All enrolled patients are stable at the time of drawing blood, and have no active asthma or exacerbation. Blood samples were collected by venipuncture from all subjects at 7:00-8:00 in the morning on an empty stomach $12 \mathrm{~h}$ after the last meal. The blood was stored in sterile PAXgene Blood RNA Tube after collection. Gently invert the PAXgene Blood RNA Tube eight- to ten-times. Store the PAXgene Blood RNA Tube upright for $12-24 \mathrm{~h}$ at room temperature $\left(18-22^{\circ} \mathrm{C}\right)$ or $4^{\circ} \mathrm{C}$. Leave at $-20^{\circ} \mathrm{C}$ for $24 \mathrm{~h}$ and finally transfer to $-80^{\circ} \mathrm{C}$ for long-term storage. PAXgene Blood RNA Tube is suitable for collecting whole blood and stabilizing RNA in cells, and can be stored for at least 50 months under $-80^{\circ} \mathrm{C}$ conditions. The blood sample was stored in the central position of the thermal container with dry ice between withdrawal and extraction. This study has been approved by the Ethics Institute of Affiliated Changzhou No.2 People's Hospital of Nanjing Medical University (2017KY024-01). The signed informed consents of all participants were obtained. This research complied with the principles of the Declaration of Helsinki.

\section{Library preparation \& high-throughput sequencing}

By using TRIzol reagent (Invitrogen, CA, USA), total RNA was extracted from blood samples according to the manual instruction. Nanodrop ND-2000 spectrophotometer (Thermo Fisher Scientific, DE, USA) was used to access the concentration and purity of RNA. The integrity of RNA was checked by using $2 \%$ agarose gel. The RNA integrity number (RIN) value was obtained by the Agilent 2100 Bioanalyzer (Agilent Technologies, CA, USA). The criteria for library construction were as follows: RNA with amount $>5 \mu \mathrm{g}$; concentration of RNA $\geq 200 \mathrm{ng} / \mathrm{ml} ; 1.8$ $<\mathrm{OD} 260 / 280<2.2$; and RIN $>7$.

First, library for high-throughput sequencing of IncRNA and mRNA was constructed as follow steps: the ribosomal RNA was removed from total RNA by Ribo-Zero Magnetic Kit (EpiCentre, WI, USA); library with Truseq RNA sample Prep Kit (Illumina, Inc., CA, USA) was constructed. The RNA was purified and fragmented into 200-500 base pairs with addition of fragmentation buffer; the first cDNA strand was synthesized by RNA fragments primed with random hexamer primers; the second cDNA strand was synthesized with dUTP instead of dTTP; after purification by Qiaquick PCR purification Kit, end repair, $3^{\prime}$ end adenylation and adapter ligation were performed; the second cDNA strand was digested by using UNG enzyme (Illumina, Inc., CA, USA) and PCR was performed to construct library for high-throughput sequencing of lncRNA and mRNA.

Second, the 18-30 nts RNA was isolated from the total RNA and then, adapter ligation and RT-PCR was performed to construct the cDNA library for high-throughput sequencing of miRNA by TruseqTM Small RNA Sample Prep Kit.

Third, all libraries for high-throughput sequencing of lncRNA and mRNA were amplified through PCR for 15 cycles. Then, purification was performed by Certified Low Range Ultra Agarose (Bio-Rad), and quantification was performed by Picogreen (Molecular Probes) on TBS380 (Turner Biosystems). Bridge PCR was performed on cBot. Sequencing was performed by a llumina HiSeq X Ten platform. 
At last, all libraries for high-throughput sequencing of miRNA were amplified through PCR for 12 cycles. Then, purification was performed by 6\% Novex TBE PAGE gel $(1.0 \mathrm{~mm}$, ten well) and quantification was performed by Picogreen (Molecular Probes) on TBS380 (Turner Biosystems). Bridge PCR was performed on cBot. Sequencing was performed by a llumina HiSeq 4000 platform.

\section{Quality control of raw sequence}

By using base calling, all the raw data obtained from high-throughput RNA-sequencing was translated into raw FASTQ sequence data. To obtain the clean reads from RNA-sequencing results for lncRNA and mRNA, sequence with low quality including adaptor sequences, sequences with quality score $<20$ and sequences with $\mathrm{N}$ base rate of raw reads $>10 \%$ were removed by using cutadapt (http://cufflinks.cbcb.umd.edu/). After sequence with low quality including adaptor sequences, sequences with quality score $<20$, sequences with $\mathrm{N}$ base and sequence less than 18bp were removed by using Fastx-Toolkit (http://hannonlab.cshl.edu/fastx_toolkit/).

\section{Clean reads mapping}

By using TopHat (http://ccb.jhu.edu/software/tophat/index.shtml), clean reads from RNA-sequencing results of mRNA and lncRNA were aligned with the human reference genome, Ensembl V84. By using Cufflinks (http: //cufflinks.cbcb.umd.edu/), the expression of lncRNA and mRNA was quantified and the normalized expression data were outputted. By using bowtie (http://bowtie-bio.sourceforge.net/index.shtml), clean reads from RNAsequencing results of miRNA were aligned with the human reference genome, GRCH38. The expression of miRNA was quantified by using miRDeep2.

\section{Identification of DEmRNAs, DEmiRNAs \& DEIncRNAs}

In RNA sequencing analysis, gene expression level is calculated by the amount of clean reads located in the genomic region. The criteria for measuring gene expression levels are RPKM (Reads Per Kilobase of exon model per Million mapped reads) and FPKM (Fragments Per Kilobase of exon model per Million mapped reads) values. Based on the alignment of all samples with the reference genome, the FPKM value of each gene in the sample is calculated, and this value is used as the expression level of the gene in the sample. Finally, the differential expression of all genes in each comparison was performed. Compared with normal control, differentially expressed mRNAs (DEmRNAs) and lncRNAs (DElncRNAs) in adult patients with asthma or severe asthma were identified by using limma package in R. Moreover, DEmRNAs and DElncRNAs between adult patients with asthma or severe asthma were identified by using limma package in $\mathrm{R}$ as well. The MRNAs and lncRNAs with p-value $<0.01$ was served as significant DEmRNAs and DElncRNAs, respectively.

Compared with normal control, differentially expressed miRNAs (DEmiRNAs) in adult patients with asthma or severe asthma were identified by using DEGseq (http://bioconductor.org/packages/DEGseq/). Furthermore, DEmiRNAs between adult patients with asthma and severe asthma were identified by using DEGseq as well. When the selection criteria of DEmiRNAs were set as $\mathrm{p}<0.01$, the same to the DEmRNAs, the amount of differentially expressed miRNA is too large to construct mRNA, lncRNA and miRNA network. Moreover, the miRNA acted as a link between mRNA and lncRNA, so it is more rigorous when screening differentially expressed miRNA, and the value of $\operatorname{logFC}$ (difference multiple) is also considered. Hence, differentially expressed miRNAs were screened according to the adjustment criteria. Hence, $p$-value $<0.001$ and $\mid \log 2$ Fold Change $\mid>2$ were defined as the criteria of significance.

Venny 2.1.0 (http://bioinfogp.cnb.csic.es/tools/venny/) was used to obtain the shared DEmRNAs, DElncRNAs and DElncRNAs in comparisons of severe asthma versus normal control, asthma versus normal control and severe asthma versus asthma.

\section{DEmiRNA-DEmRNA interaction analysis}

DEmiRNA-DEmRNA interaction pairs in severe asthma versus normal control, asthma versus normal control and severe asthma versus asthma were obtained. As miRNAs tend to decrease the expression of their target mRNA, target genes were selected from DEmRNAs that expressed inversely with that of miRNA, to subject to further investigation. First, the putative targeted DEmRNAs of DEmiRNAs were predicted by six bioinformatic algorithms (RNA22, miRanda, miRDB, miRWalk, PICTAR2 and Targetscan). Then, the confirmed targeted DEmRNAs of DEmiRNAs were obtained from miRWalk. Third, the confirmed DEmiRNA-DEmRNA pairs were derived from miRWalk and the DEmiRNA-DEmRNA pairs recorded by $\geq 4$ algorithms. Based on the obtained DEmiRNA- 
DEmRNA pairs, DEmiRNA-DEmRNA interaction networks in severe asthma versus normal control, asthma versus normal control and severe asthma versus asthma were constructed by using Cytoscape software. Shared DEmiRNA-DEmRNA pairs in both asthma and severe asthma compared with normal controls were obtained as well.

\section{DEmiRNA-DEIncRNA interaction analysis}

DEmiRNA-DElncRNA interaction pairs in severe asthma versus normal control, asthma versus normal control and severe asthma versus asthma were obtained. MiRNA may negatively regulate the expression of IncRNA through the mechanism similar to mRNA. So DEmiRNA-DElncRNA pairs in which DElncRNAs were expressed inversely with DEmiRNAs were retained for following research. The putative targeted DElncRNAs of DEmiRNAs were predicted by five bioinformatic algorithms including miRWalk, miRanda, PITA, RNAhybrid and Targetscan. Based on the obtained DEmiRNA-DElncRNA pairs, DEmiRNA-DElncRNA interaction networks in severe asthma versus normal control, asthma versus normal control and severe asthma versus asthma were constructed by using Cytoscape software. Shared DEmiRNA-DElncRNA pairs in both asthma and severe asthma compared with normal controls were obtained as well.

\section{DEmRNA-DElncRNA interaction analysis}

Previous study has indicated that lncRNAs could play a cis-regulatory role on the expression of genes that transcribed near them [14]. The DEmRNAs transcribed within a $10 \mathrm{~kb}$ window up- or $100 \mathrm{~kb}$ down-stream of DElncRNAs were searched and served as nearby DEmRNAs of DElncRNAs in this present study. Based on the DEmRNAs and DElncRNAs derived from shared DEmiRNA-DEmRNA pairs and DEmiRNA-DElncRNA pairs in both asthma and severe asthma compared with normal controls, DElncRNA-nearby DEmRNA pairs were obtained. In addition, DElncRNA-nearby DEmRNA pairs were searched based on the DEmRNAs and DElncRNAs derived from DEmiRNA-DEmRNA pairs and DEmiRNA-DElncRNA pairs between severe asthma and asthma.

Moreover, DEmRNAs coexpressed with DElncRNAs were identified as well. The pairwise Pearson correlation coefficients between DElncRNAs and DEmRNAs were calculated. DElncRNA-DEmRNA pairs with $\mathrm{p}<0.001$ and $|r| \geq 0.8$ were served as significant mRNA-IncRNA coexpression pairs. DElncRNA-DEmRNA coexpression pairs in severe asthma versus normal control, asthma versus normal control and severe asthma versus asthma were obtained. Moreover, shared DElncRNA-DEmiRNA coexpression pairs in both asthma and severe asthma compared with normal controls were obtained as well. DElncRNA-DEmRNA coexpression networks were constructed by using Cytoscape software (http://www.cytoscape.org/).

\section{DEmiRNA-DEmRNA-DElncRNA interaction network}

Based on the shared DEmiRNA-DEmRNA pairs, DEmiRNA-DElncRNA pairs and DEmRNA-DElncRNA interaction pairs in both asthma and severe asthma compared with normal controls, the shared DEmiRNADEmRNA-DElncRNA interaction network was constructed.

Based on the DEmiRNA-DEmRNA pairs, DEmiRNA-DElncRNA pairs and DEmRNA-DElncRNA interaction pairs between severe asthma and asthma, the severe asthma-specific DEmiRNA-DEmRNA-DElncRNA interaction network was constructed.

\section{Functional analysis of DEmRNAs}

Gene ontology (GO) and Kyoto Encyclopedia of Genes and Genomes (KEGG) molecular pathway enrichment analysis of DEmRNAs derived from the shared and severe asthma-specific DEmiRNA-DEmRNA-DElncRNA were performed by using online-based software GeneCoDis3 (http://genecodis.cnb.csic.es/analysis), respectively. Hierarchical clustering analysis of DEmRNAs were conducted by using 'pheatmap' in R version 3.3.3.

\section{Validation in the Gene Expression Omnibus dataset}

GSE69683 dataset was downloaded from the Gene Expression Omnibus (GEO; www.ncbi.nlm.nih.gov/geo/), which consisted of 344 patients with severe asthma and 87 normal controls. The dataset GSE69683 examined the blood sample. The expression patterns of selected DEmRNAs were validated in GSE69683 dataset. 


\begin{tabular}{|c|c|c|c|c|c|c|}
\hline Symbol & \multicolumn{2}{|c|}{ Asthma vs control } & \multicolumn{2}{|c|}{ Severe asthma vs control } & \multicolumn{2}{|c|}{ Severe asthma vs asthma } \\
\hline \multicolumn{7}{|l|}{ IncRNA } \\
\hline RP5-857K21.7 & -0.98 & $3.10 \mathrm{E}-03$ & -2.05 & $2.41 \mathrm{E}-04$ & -1.07 & $8.18 \mathrm{E}-03$ \\
\hline RP11-58A11.2 & 0.67 & $1.13 E-03$ & 0.42 & $7.39 \mathrm{E}-03$ & -0.24 & $1.80 \mathrm{E}-04$ \\
\hline \multicolumn{7}{|l|}{ mRNA } \\
\hline PABPC1 & -0.84 & $4.33 \mathrm{E}-04$ & -1.38 & $1.09 \mathrm{E}-04$ & -0.54 & $9.85 \mathrm{E}-03$ \\
\hline PIP5K1B & 0.53 & $3.58 \mathrm{E}-04$ & 0.91 & $2.61 E-04$ & 0.38 & $9.56 \mathrm{E}-03$ \\
\hline USP53 & 1.35 & $9.32 \mathrm{E}-04$ & 0.50 & $8.45 E-03$ & -0.86 & $8.06 \mathrm{E}-03$ \\
\hline SNRPE & 1.31 & $3.21 \mathrm{E}-04$ & 0.69 & $5.35 \mathrm{E}-03$ & -0.62 & $6.47 \mathrm{E}-03$ \\
\hline hsa-miR-98-5p & -2.77 & $<1.00 \mathrm{E}-16$ & 2.06 & $<1.00 \mathrm{E}-16$ & 4.83 & $<1.00 \mathrm{E}-16$ \\
\hline hsa-miR-18a-5p & -2.01 & $1.54 \mathrm{E}-13$ & 2.21 & $<1.00 \mathrm{E}-16$ & 4.22 & $<1.00 \mathrm{E}-16$ \\
\hline
\end{tabular}

\section{Results}

The characteristics of all subjects for analysis

The clinical data of all subjects was displayed in Table 1. All subjects have no history of smoking. The mean age of three patients with asthma, three patients with severe asthma and three normal controls were 52, 53 and 51, respectively. Each group consists of two women and one man. All nine persons belong to Asians from China.

\section{Analysis of transcriptome sequencing}

We sequenced RNA libraries of nine blood samples from three patients with asthma, three patients with severe asthma and three normal controls. Counts of clean reads of sequencing results were displayed in Supplementary Table 1.

\section{Identification of DEmRNAs, DEmiRNAs \& DEIncRNAs}

A total of 505 DElncRNAs (20 upregulated and 76 downregulated DElncRNAs), 608 DElncRNAs (504 upregulated and 104 downregulated DElncRNAs) and 143 DElncRNAs (50 upregulated and 93 downregulated DElncRNAs) were identified in asthma versus normal controls, severe asthma versus normal controls and severe asthma versus asthma, respectively (Figure 1A). Moreover, a total of 217 shared DElncRNAs were identified in both asthma and severe asthma compared with normal controls (Figure 1B).

A total of 962 DEmRNAs (576 upregulated and 386 downregulated DEmRNAs), 1328 DEmRNAs (780 upregulated and 548 downregulated DEmRNAs) and 95 DEmRNAs (17 upregulated and 78 downregulated DEmRNAs) were identified in asthma versus normal controls, severe asthma versus normal controls and severe asthma versus asthma, respectively (Figure 1C). Moreover, a total of 487 shared DEmRNAs were identified in both asthma and severe asthma compared with normal controls (Figure 1D).

A total of 53 DEmiRNAs (18 upregulated and 35 downregulated DEmiRNAs), 63 DEmiRNAs (61 upregulated and two downregulated DEmiRNAs) and 96 DEmiRNAs (88 upregulated and eight downregulated DEmiRNAs) were identified in asthma versus normal controls, severe asthma versus normal controls and severe asthma versus asthma, respectively (Figure 1E). Moreover, a total of 13 shared DEmiRNAs were identified in both asthma and severe asthma compared with normal controls (Figure 1F).

Shared DEmRNAs, DElncRNAs and DEmiRNAs in asthma versus normal controls, severe asthma versus normal controls and severe asthma versus asthma were displayed in Table 2. 


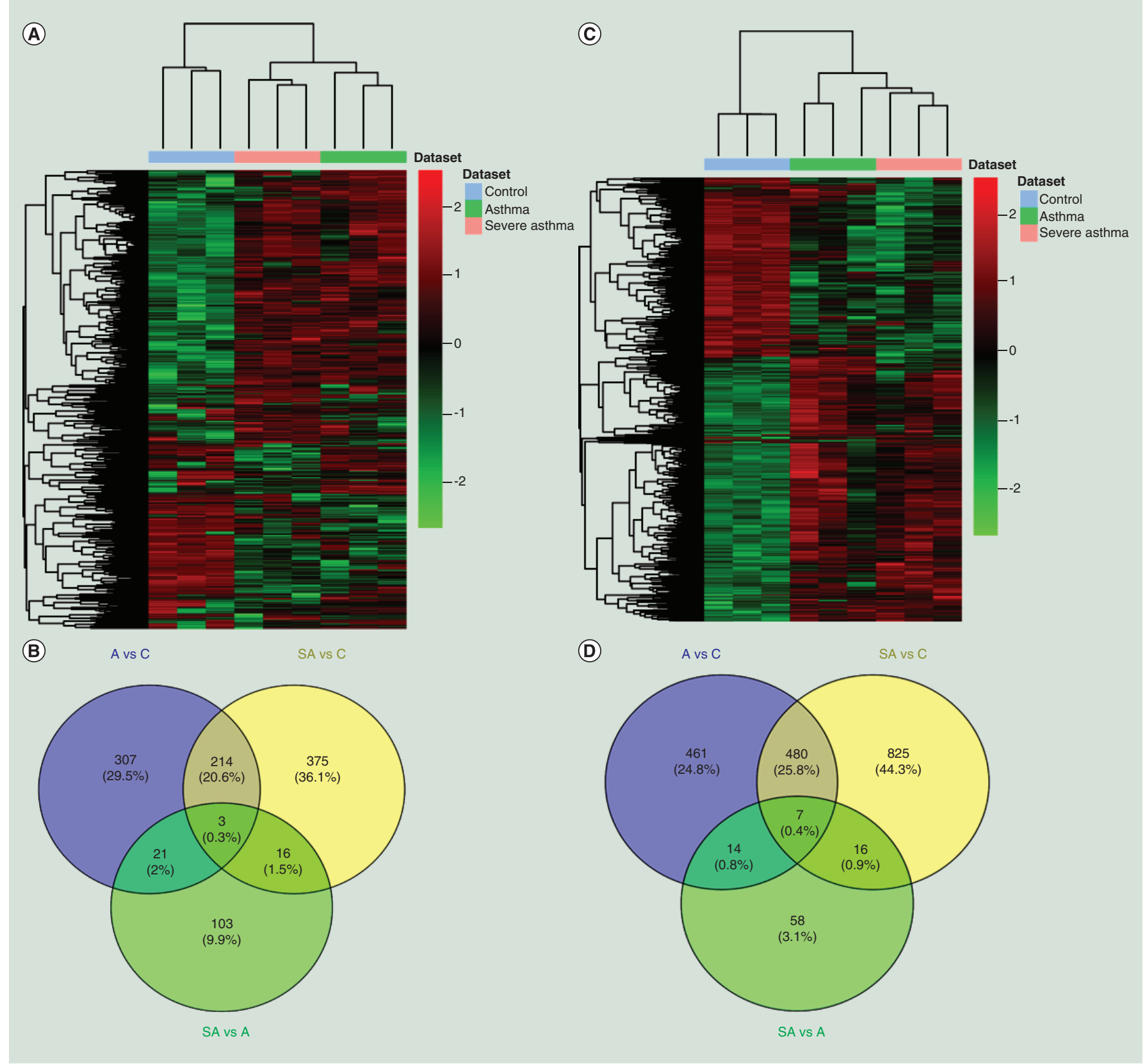

Figure 1. Differentially expressed IncRNAs, mRNAs and miRNAs in normal control, asthma and severe asthma (SA). (A, C \& E) were hierarchical clustering analysis of DEIncRNAs, DEmRNAs and DEmiRNAs in normal control, asthma and severe asthma. Row represented DEIncRNAs, DEmRNAs and DEmiRNAs. Column represented samples. The color scale represented the expression levels. (B, D \& F) shown the venny analysis of DEmRNAs, DElncRNAs and DEIncRNAs in severe asthma versus normal control (SA vs C), asthma versus normal control ( $A$ vs $C$ ) and severe asthma versus asthma (SA vs $A$ ).

A:Asthma; C: Control; SA: Severe asthma.

\section{DEmiRNA-DEmRNA interaction}

A total of 653, 1056 and 410 DEmiRNA-DEmRNA interaction pairs were identified in asthma versus normal controls, severe asthma versus normal controls and severe asthma versus asthma. Based on these 410 DEmiRNADEmRNA interaction pairs in severe asthma versus asthma, the severe asthma-specific DEmiRNA-DEmRNA interaction network was constructed. The RAPH1 (degree $=28)$, BCL11B (degree $=27)$ and SNX1 (degree $=26)$ were three hub genes of the severe asthma-specific DEmiRNA-DEmRNA interaction network (Supplementary Figure 1A). MiR-93-5p (degree = 15), miR-3613-3p (degree = 15) and miR-15b-5p (degree = 13) were three hub 
(E)
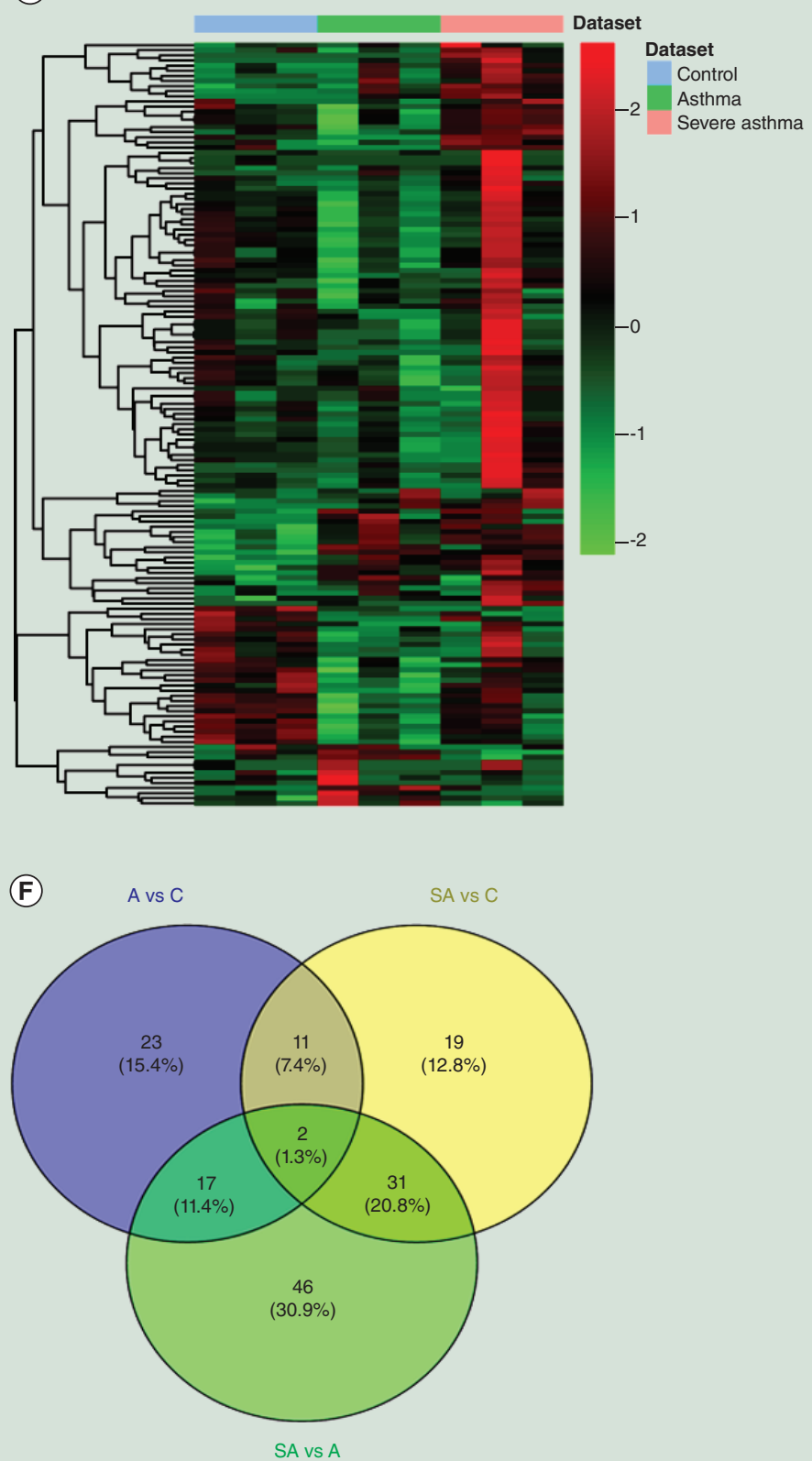

Figure 1. Differentially expressed IncRNAs, mRNAs and miRNAs in normal control, asthma and severe asthma (SA) (cont.). (A, C \& E) were hierarchical clustering analysis of DEIncRNAs, DEmRNAs and DEmiRNAs in normal control, asthma and severe asthma. Row represented DEIncRNAs, DEmRNAs and DEmiRNAs. Column represented samples. The color scale represented the expression levels. (B, D \& F) shown the venny analysis of DEmRNAs, DElncRNAs and DEIncRNAs in severe asthma versus normal control (SA vs C), asthma versus normal control (A vs $C$ ) and severe asthma versus asthma (SA vs A).

A:Asthma; C: Control; SA: Severe asthma.

miRNAs of this network. Moreover, a total of 34 shared DEmiRNA-DEmRNA interaction pairs were identified in both asthma and severe asthma compared with normal controls. MiR-122-5p (degree $=10)$ and miR-133a-3p $($ degree $=7)$ were two hub miRNAs of this shared DEmiRNA-DEmRNA interaction network (Supplementary Figure 1B). 


\begin{tabular}{|c|c|c|c|}
\hline KEGG ID & KEGG pathways & FDR & Genes \\
\hline \multicolumn{4}{|c|}{ Shared DEmRNAs in both asthma and severe asthma } \\
\hline Kegg:04666 & Fc gamma R-mediated phagocytosis & 0.013354 & ARPC5, RAC1 \\
\hline Kegg:04810 & Regulation of actin cytoskeleton & 0.014753 & ARPC5, MSN, RAC1 \\
\hline Kegg:05200 & Pathways in cancer & 0.013003 & SPI1, TGFB1, RAC1 \\
\hline Kegg:04670 & Leukocyte transendothelial migration & 0.017984 & MSN, RAC1 \\
\hline Kegg:05211 & Renal cell carcinoma & 0.010012 & TGFB1, RAC1 \\
\hline Kegg:05210 & Colorectal cancer & 0.013772 & TGFB1, RAC1 \\
\hline Kegg:05212 & Pancreatic cancer & 0.010012 & TGFB1, RAC1 \\
\hline Kegg:04380 & Osteoclast differentiation & 0.006689 & SPI1, TGFB1, RAC1 \\
\hline Kegg:05100 & Bacterial invasion of epithelial cells & 0.010012 & ARPC5, RAC1 \\
\hline Kegg:05131 & Shigellosis & 0.01778 & ARPC5, RAC1 \\
\hline \multicolumn{4}{|c|}{ Severe-asthma-specific DEmRNAs } \\
\hline KEGG ID & KEGG pathways & Hyp_c & Genes \\
\hline Kegg:03040 & Spliceosome & 0.028807 & SF3A1, SNRPE, SF3B4 \\
\hline
\end{tabular}

\section{DEmiRNA-DEIncRNA interaction network}

A total of 1147, 601 and 890 DEmiRNA-DElncRNA interaction pairs were identified in asthma versus normal controls, severe asthma versus normal controls and severe asthma versus asthma. Based on these 890 DEmiRNADElncRNA interaction pairs in severe asthma versus asthma, the severe asthma-specific DEmiRNA-DElncRNA interaction network was constructed (Supplementary Figure 2A). AP000569.8 (degree = 68), C14orf64 (degree = 47) and AC010976.2 (degree = 46) were three hub IncRNAs of the severe asthma-specific DEmiRNA-DElncRNA interaction network. MiR-1827 (degree $=22)$, miR-491-5p (degree $=20)$ and miR-378g (degree $=19)$ were three hub miRNAs of this network. Moreover, a total of 25 shared DEmiRNA-DElncRNA interaction pairs were identified in both asthma and severe asthma compared with normal controls (Supplementary Figure 2B). MiR3130-5p (degree $=6$ ) and miR-122-5p (degree = 6) were two hub miRNAs of this shared DEmiRNA-DElncRNA interaction network. RP11-361M10.5 (degree $=5$ ) was the hub lncRNA of this network.

\section{DEIncRNA-DEmRNA interaction network}

A total of 64 DElncRNAs and 54 DEmRNAs were obtained from the severe asthma-specific DEmiRNADElncRNA and DEmiRNA-DEmRNA interaction network, respectively. Based on these 64 DElncRNAs and 54 DEmRNAs, two DElncRNA-nearby DEmRNA pairs (AC005237.4-PASK and CTD-2376I20.1-EVL) and 184 DElncRNA-DEmRNA coexpression pairs were identified (Supplementary Figure 3A).

A total of 17 DElncRNAs and 27 DEmRNAs were obtained from the shared DEmiRNA-DElncRNA and DEmiRNA-DEmRNA interaction network in both asthma and severe asthma, respectively. Based on these 17 DElncRNAs and 27 DEmRNAs, no DElncRNA-nearby DEmRNA pairs were identified while 13 DElncRNADEmRNA coexpression pairs were identified (Supplementary Figure 3B).

Shared DEmiRNA-DEmRNA-DEIncRNA interaction network in both asthma \& severe asthma The shared DEmiRNA-DEmRNA-DElncRNA interaction network in both asthma and severe asthma was consisted of ten DEmiRNAs, 17 DElncRNAs and 27 DEmRNAs (Figure 2A). MiR-133a-3p (degree = 8), miR-517a-3p $($ degree $=4)$ and miR-517b-3p (degree $=4)$ were three hub miRNAs. Hierarchical clustering analysis of these ten DEmiRNAs, 17 DElncRNAs and 27 DEmRNAs were displayed in Figure 2B . Subnetwork of miR-133a-3p and expression of miR-133a-3p-EFHD2/CNN2-AC144831.1 interactions in normal control, asthma and severe asthma were displayed in Figure $4 \mathrm{~A} \& \mathrm{~B}$.

Based on the functional annotation of these 27 DEmRNAs derived from shared DEmiRNA-DEmRNADElncRNA interaction network, cytoskeleton organization (false discovery rate $[\mathrm{FDR}]=1.59 \mathrm{E}-02$ ), inflammatory response $(\mathrm{FDR}=1.93 \mathrm{E}-02)$, protein binding $(\mathrm{FDR}=1.15 \mathrm{E}-05)$ and nucleus $(\mathrm{FDR}=9.57 \mathrm{E}-04)$ were significantly enriched GO terms (Supplementary Table 2). Based on the KEGG enrichment analysis, Fc gamma R-mediated phagocytosis $(\mathrm{FDR}=0.0133538)$ was a significantly enriched pathways $($ Table 3$)$. 
(A)
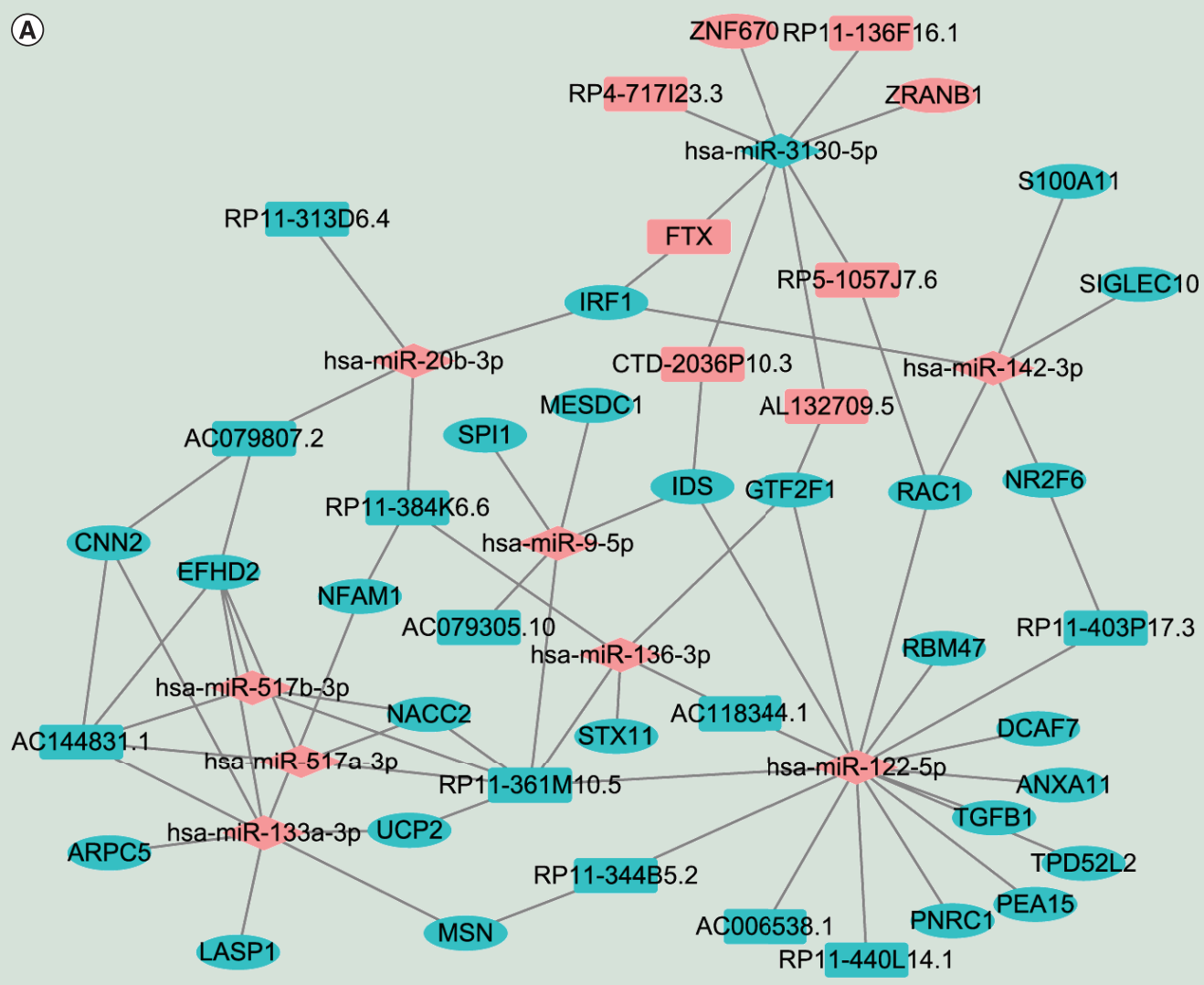

Figure 2. Shared differentially expressed miRNA-mRNA-IncRNA interaction network in both asthma and severe asthma. (A) Shared DEmiRNA-DEmRNA-DEIncRNA interaction network in both asthma and severe asthma. The rectangles, ellipses and rhombuses represented DEmiRNAs, DEmRNAs and DElncRNAs, respectively. Red and green color represented up and downregulation, respectively. (B) Hierarchical clustering analysis of DElncRNAs, DEmRNAs and DEmiRNAs derived from this network in normal control, asthma and severe asthma. Row represented DEIncRNAs, DEmRNAs and DEmiRNAs. Column represented samples.

Severe asthma-specific DEmiRNA-DEmRNA-DEIncRNA interaction network

The severe asthma-specific DEmiRNA-DEmRNA-DElncRNA interaction network was consisted of 196 DEmiRNAs, 139 DElncRNAs and 95 DEmRNAs (Figure 3A). MiR-3613a-3p (degree = 31), miR1827 (degree $=30)$ and miR-93-5p (degree $=28)$ and miR-15b-5p (degree $=28)$ were four hub miRNAs. Hierarchical clustering analysis of these 196 DEmiRNAs, 139 DElncRNAs and 95 DEmRNAs were displayed in Figure 3B. Subnetwork of miR-3613-3p and miR-93-5p and expression of miR3613-3p-CD44/BCL11B-LINC00158/CTA-217C2.1/AC010976.2/RP11-641A6.2 interactions and miR-935p-BCL11B-LINC00158/CTA-217C2.1/AC010976.2/RP11-641A6.2 interactions in normal control, asthma and serve asthma were displayed in Figure 4C-F.

Based on the functional annotation, regulation of transcription, DNA-dependent $(F D R=0.0020768)$, metal ion binding $(F D R=0.0322671)$, nucleus ( $F D R=0.000048193)$ were significantly enriched GO terms (Supplementary Table 3). Based on the KEGG enrichment analysis, Spliceosome (FDR $=0.028807$ ) was the only one significantly enriched pathway (Table 3).

\section{Validation in GEO dataset}

The expression patterns of selected DEmRNAs (SNRPE, SF3B4, RAC1, PABPC1, C12orf55, BCL11B and CD44) were verified using GSE69683 dataset. The dataset GSE69683 consisted of 334 patients with severe asthma and 87 normal controls, which were all from the USA. The dataset GSE69683 examined the blood sample. As shown in Figure 5, BCL11B was downregulated and CD44 was upregulated, which was inconsistent with 


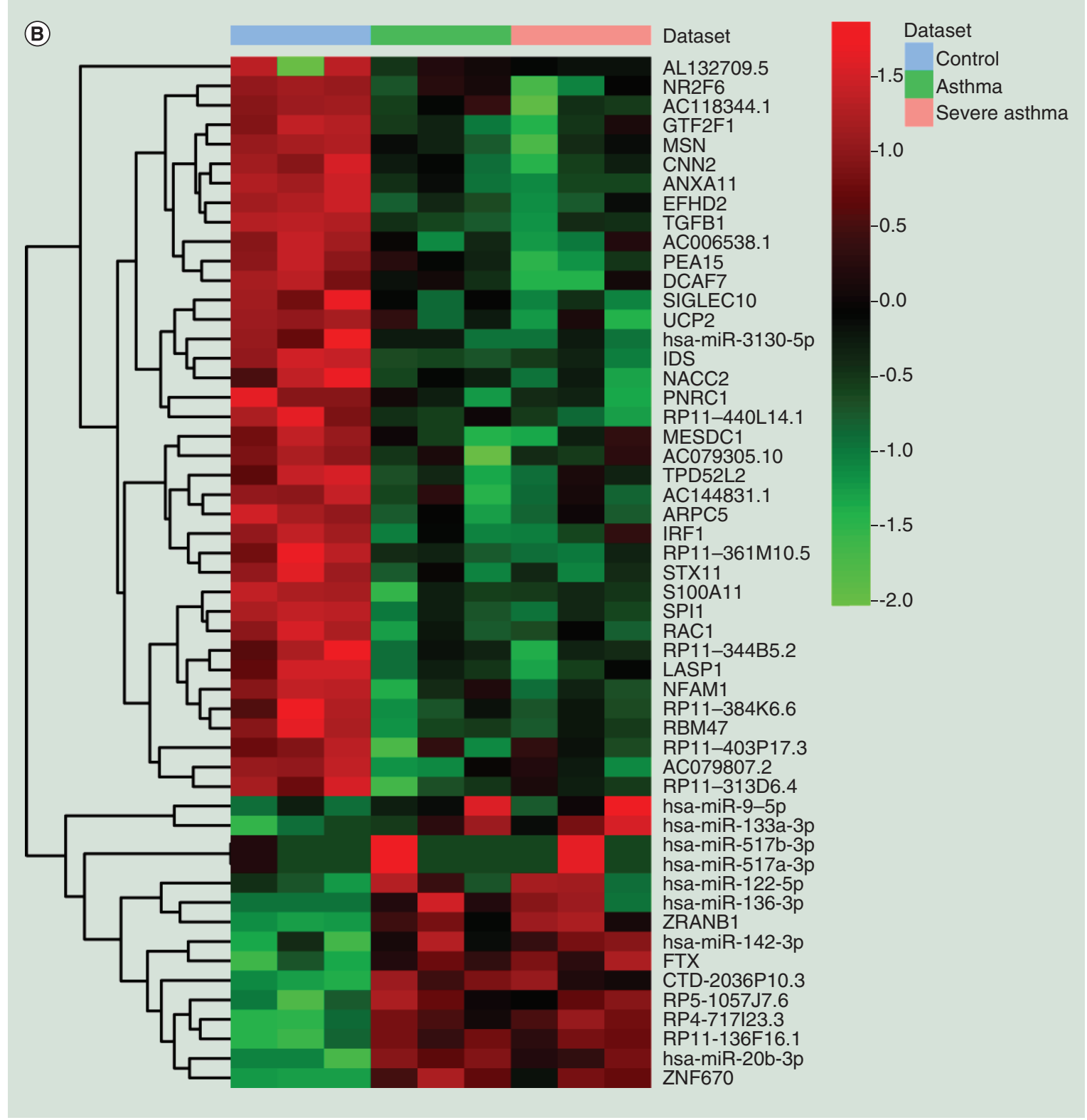

Figure 2. Shared differentially expressed miRNA-mRNA-IncRNA interaction network in both asthma and severe asthma (cont.). (A) Shared DEmiRNA-DEmRNA-DEIncRNA interaction network in both asthma and severe asthma. The rectangles, ellipses and rhombuses represented DEmiRNAs, DEmRNAs and DEIncRNAs, respectively. Red and green color represented up and downregulation, respectively. (B) Hierarchical clustering analysis of DEIncRNAs, DEmRNAs and DEmiRNAs derived from this network in normal control, asthma and severe asthma. Row represented DEIncRNAs, DEmRNAs and DEmiRNAs. Column represented samples.

our RNA-sequencing results. But for most of the selected DEmRNAs, the expression was consistent with our RNA-sequencing results, such as SF3B4, RAC1, PABPC1, SNRPE and C12orf55. The SF3B4, RAC1 and PABPC1 were downregulated, while $S N R P E$ and $C 12$ orf 55 were upregulated in severe asthma suggesting that the results of RNA-sequencing were convincing.

\section{Discussion}

Severe asthma is a complex disease affecting millions of individuals worldwide, and is increasingly recognized as a major unmet need [7]. Although severe asthma accounts for only a minority of patients with asthma, it contributes 


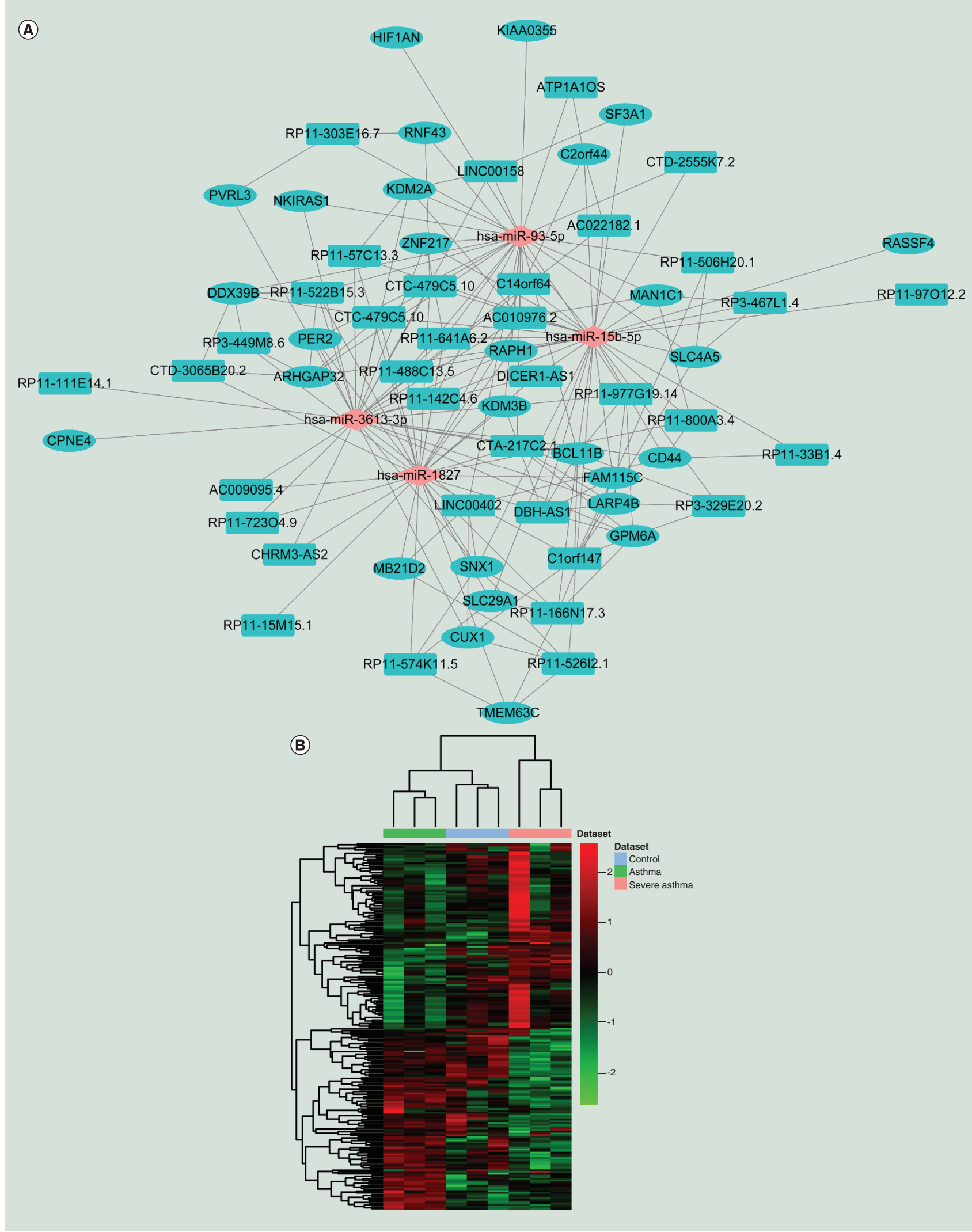

Figure 3. Severe asthma-specific differentially expressed miRNA-mRNA-IncRNA interaction network in both asthma and severe asthma. (A) Severe asthma-specific DEmiRNA-DEmRNA-DEIncRNA interaction network in both asthma and severe asthma. The rectangles, ellipses and rhombuses represented DEmiRNAs, DEmRNAs and DEIncRNAs, respectively. Red and green color represented up and downregulation, respectively. (B) Hierarchical clustering analysis of DEIncRNAs, DEmRNAs and DEmiRNAs derived from this network in normal control, asthma and severe asthma. Row represented DEIncRNAs, DEmRNAs and DEmiRNAs. Column represented samples.

DEIncRNA: Differentially expressed IncRNA; DEmRNA: Differentially expressed mRNA; DEmiRNA: Differentially expressed miRNA. 
(A)

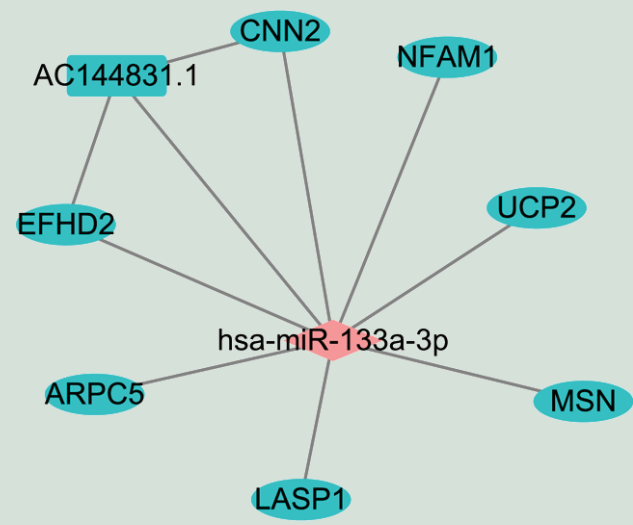

(C)

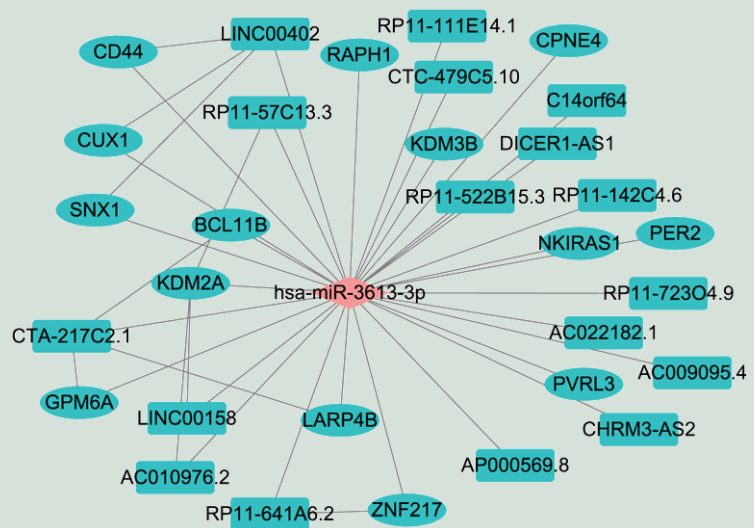

(E)

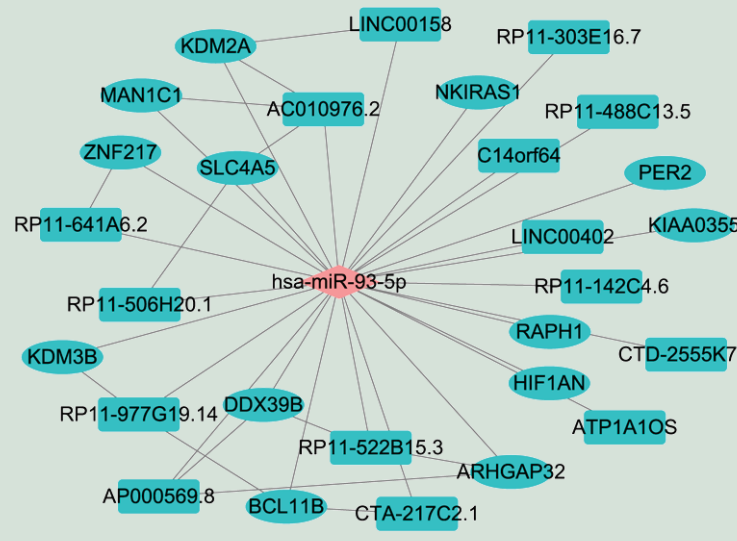

(B)

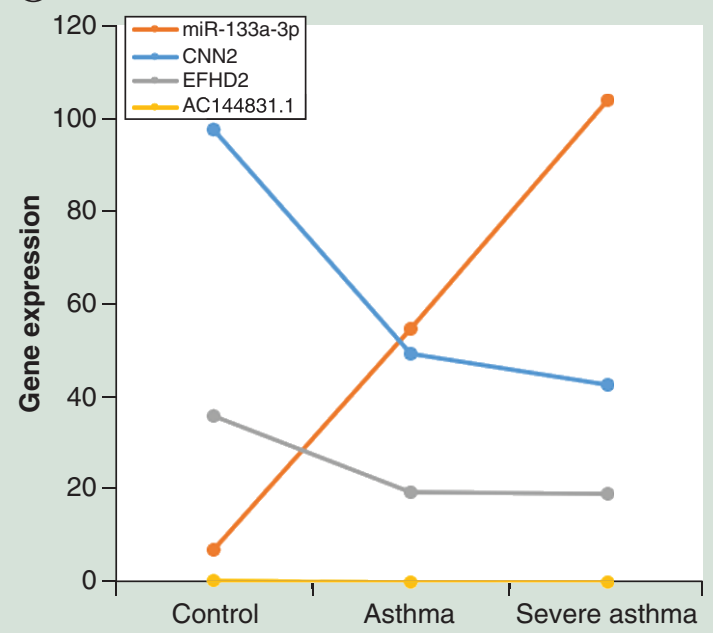

(D)

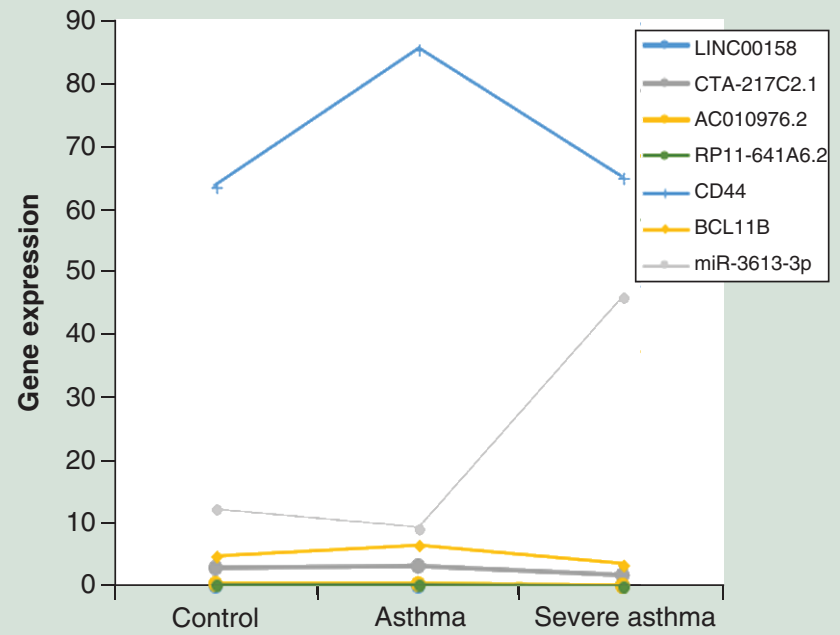

(F)

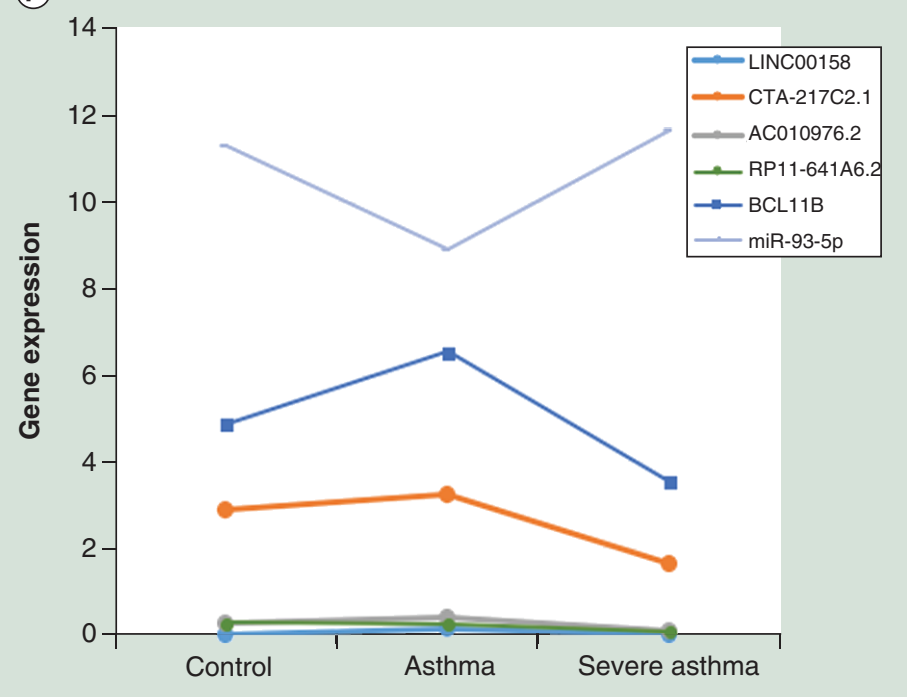

Figure 4. Severe asthma-specific and shared DEmiRNA-DEmRNA-DEIncRNA interactions. (A, C \& E) were subnetworks of miR-133a-3p, miR-3613-3p and miR-93-5p. The rectangles, ellipses and rhombuses represented DEmiRNAs, DEmRNAs and DElncRNAs, respectively. Red and green color represented up and downregulation, respectively. (B, D \& F) were expression of miR-133a-3p-EFHD2/CNN2-AC144831.1, miR-3613-3p-CD44/ BCL11B-LINC00158/CTA-217C2.1/AC010976.2/RP11-641A6.2 and miR-93-5p- BCL11B-LINC00158/CTA-217C2.1/AC010976.2/RP11-641A6.2 interactions in normal control, asthma and severe asthma. DEIncRNA: Differentially expressed IncRNA; DEmRNA: Differentially expressed mRNA; DEmiRNA: Differentially expressed miRNA. 


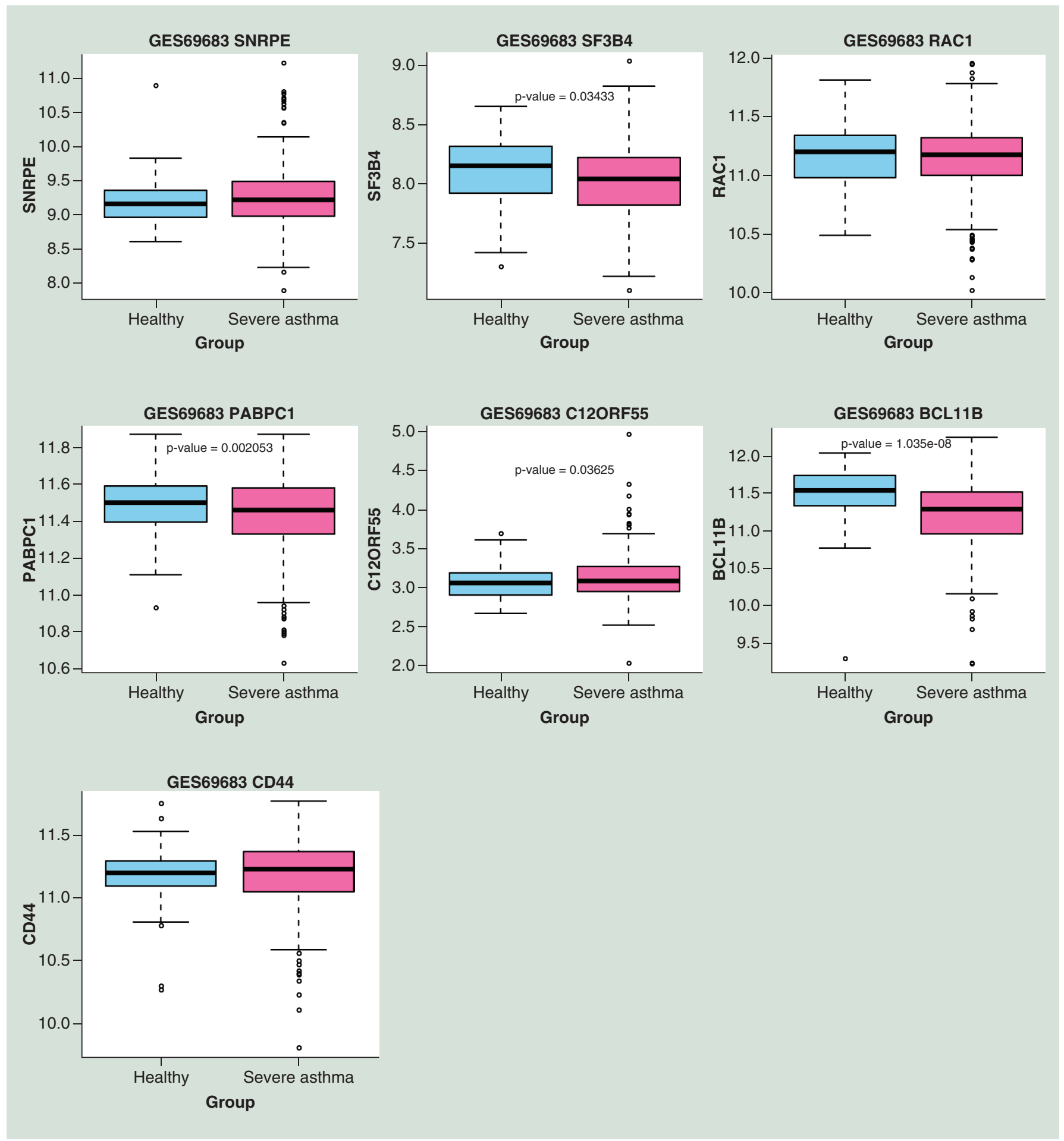

Figure 5. Validation of selected differentially expressed mRNAs in GSE69683. The x-axis shows healthy normal control (blue colour) and severe asthma (red colour) groups and $y$-axis shows a log2 transformation to the intensities.

to the majority of the morbidity, mortality and economic costs of the disease. However, the molecular mechanism of severe asthma remains unclear.

To better understanding the pathogenesis and developing potential biomarkers of asthma and severe asthma, this present study utilized next-generation sequencing to provide a quantitative and comprehensive analysis of the 
coding and noncoding transcriptome in asthma and severe asthma. These analyses revealed significant differences in the patterns of miRNA, IncRNA and mRNA expression in asthma and severe asthma, as well as the dynamic changes of DEmiRNAs, DElncRNAs and DEmRNAs. Generally, our data suggest that distinct populations of miRNAs, lncRNAs and mRNAs are involved in the pathogenesis of asthma and severe asthma. Recent years, ncRNAs have been found to be associated with wide range of biological regulatory functions [15]. However, most of the DElncRNAs obtained in this study were with unknown function, which is mainly due to the few researches for them. Hence, shared and severe asthma-specific DEmiRNA-DEmRNA-DElncRNA interaction networks were constructed. In these networks, DEmRNAs were coexpressed with DElncRNAs, and both DEmRNAs and DElncRNAs were targets of DEmiRNAs.

A total of 13 shared DEmiRNAs, 217 shared DElncRNAs and 487 shared DEmRNAs were identified in both asthma and severe asthma compared with normal controls. The shared and severe asthma-specific DEmiRNADEmRNA-DElncRNA interaction network in both asthma and severe asthma were consisted of ten DEmiRNAs, 17 DElncRNAs and 27 DEmRNAs. MiR-133a-3p-EFHD2/CNN2-AC144831.1 interactions involve with both asthma and severe asthma. Upregulated miR-133a-3p and its downregulated targets including EFHD2, CNN2 and IncRNA, AC144831.1 were first found in both asthma and severe asthma compared with normal controls.

It is reported that miR-133a-3p might be regulated by IL-17 [16]. The IL-17-producing T cells has key role in asthma, which was upregulated in both patients with asthma [17,18] and animal models of asthma [19]. The IL-17A response may be variable during the development of allergic airway inflammation in asthma animal models and be affected by experimental conditions, such as sensitization procedure, dosing pathways, antigenic doses, sampling time, etc [20-23]. In fact, a clinical trial demonstrated the high-dose and low-responder subpopulations in a therapeutic effect of brodalumab, a human anti-IL17RA monoclonal antibody, which inhibits the biologic activity of IL-17A, in patients with inadequately controlled moderate to severe asthma [24]. Chiba et al. reported that IL-17A upregulates the RhoA protein through downregulation of miR-133a-3p, which is one of the causes of asthmatics [16]. Therefore, we presumed that IL-17 might involve in the development of asthma by regulating miR133a-3p. Although, no previous study reported that the association between EF-hand domain family member D2 (EFHD2) and asthma, EFHD2 and CNN2 shared with the same binding miRNA, miR-133a-3p which suggested that EFHD2 might play a role in the process of both asthma and severe asthma by interacting with miR-133a3p- CNN2. AC144831.1 was a target of miR-133a-3p that coexpressed with both EFHD2 and CNN2 which suggested that miR-133a-3p-EFHD2/CNN2-AC144831.1 interactions play an important role in the occurrence and development of asthma and severe asthma.

To better research the mechanism of severe asthma, we constructed the severe asthma-specific DEmiRNADEmRNA-DElncRNA interaction network. The expression of miR-3613-3p-CD44/BCL11B interaction was significantly altered in severe asthma compared with asthma. The $C D 44$ is a major hyaluronan-binding protein and decreased on alveolar macrophages was reported to involve with deranged hyaluronan homeostasis is in asthma [25]. In addition, accumulated evidence showed that toll-like receptor (TLR) signaling plays an important role in innate immunity of asthma and CD44 serves as a negative regulator of TLR signaling [17,18,26]. Decreased expression of CD44 could induce impaired hyaluronan clearance and upregulate TLR2 and TLR4 on macrophages which resulted in persistent inflammation [25]. In this present study, CD44 was significantly downregulated in severe asthma compared with asthma which provided evidence for previous studies and highlighted the importance of CD44 in severe asthma.

BCL11B is expressed in thymocytes which are critical for Type-2 T-helper (Th2) lineage licensing and the development of T-regulatory cells [27]. Th2 cells are formed following the activation of naive $\mathrm{CD} 4^{+} \mathrm{T}$ cells in the presence of IL-4, and are critical in allergic diseases such as asthma [19]. Downregulated BCL11B was found in severe asthma compared with asthma.

Correspondingly, miR-3613-3p was significantly upregulated in severe asthma compared with asthma which suggested that miR-3613-3p plays a role in severe asthma by regulating both CD44 and BCL11B. Four targets (LINC00158, CTA-217C2.1, AC010976.2 and RP11-641A6.2) of miR-3613-3p that coexpressed with both $C D 44$ and $B C L 11 B$ were first found to be aberrant expressed between severe asthma and asthma. Hence, we speculated that miR-3613-3p-CD44/BCL11B-LINC00158/CTA-217C2.1/AC010976.2/RP11-641A6.2 interactions contributed to the development of asthma to severe asthma.

In addition, BCL11B and the above four IncRNAs (LINC00158, CTA-217C2.1, AC010976.2 and RP11641A6.2) were shared targets of another severe asthma-specific miRNA, miR-93-5p. Downregulated miR-93 in the CD3(+) IL-22(+) compared with CD3(+)IL-22(-) T cells which was speculated to impact the T-cell 
responses in asthma [28]. These finding emphasized the importance of miR-93-5p-BCL11B-LINC00158/CTA217C2.1/AC010976.2/RP11-641A6.2 interactions in the development of severe asthma.

Function annotation of DEmRNAs derived from the shared DEmiRNA-DEmRNA-DElncRNA interaction network in both asthma and severe asthma indicated that $\mathrm{Fc}_{\mathrm{c}} \gamma \mathrm{R}$-mediated phagocytosis involve with asthma and development of severe asthma. Spliceosome was the only one enriched pathway according to function annotation of DEmRNAs derived from severe asthma-specific DEmiRNA-DEmRNA-DElncRNA interaction network, which suggested that spliceosome and three spliceosome-related genes (SF3A1, SNRPE and SF3B4) involve with the development of severe asthma.

In conclusion, we identified the shared and diverging genes, IncRNAs, miRNAs and signaling pathways in asthma and severe asthma. Moreover, the network of IncRNA-miRNA-mRNA interactions will facilitate further experimental studies and may be used to refine biomarker predictions for developing novel therapeutic approaches in severe asthma.

\section{Limitation}

There is a limitation of our study. The small sample size (three sample per group) for RNA-sequencing might influence the quality of our results. Although the validation based on GSE69683 suggested that our RNA-sequencing results were generally convincing, studies with larger sample size need to be conducted to confirm this conclusion. Moreover, further experiment was needed to solve the biological significances of important mRNAs, IncRNAs and miRNAs in severe asthma.

\section{Future perspective}

Our study determined the DEmRNA, DEmiRNA and DElncRNA expression data from normal controls, asthma and severe asthma by using RNA sequencing. MiR-133a-3p-EFHD2/CNN2AC144831.1 interactions might play an important role in both asthma and severe asthma. MiR-36133p-CD44/BCL11B-LINC00158/CTA-217C2.1/AC010976.2/RP11-641A6.2 interactions and miR-93-5p$B C L 11 B$-LINC00158/CTA-217C2.1/AC010976.2/RP11-641A6.2 interactions were speculated to involve with the development of asthma to severe asthma. This study provides clues for understanding the mechanisms of severe asthma and developing novel therapeutic strategies for severe asthma. In the future, we plan to first validate the expression of the identified key mRNAs, IncRNAs and miRNAs in a larger-scale study, and further investigate the biological significances of important mRNAs, lncRNAs and miRNAs in model systems or cell lines. These experiments may contribute to understanding the pathological mechanism of severe asthma and finding potential drug targets for severe asthma therapy.

Financial \& competing interests disclosure

This work was supported by Jiangsu province 'six big talent peak' project (grant number WSN092, Q Zhang), Changzhou HighLevel Medical Talents Training Project (grant number 2016CZLJ017, Q Zhang) and Jiangsu province '333 talents' project (grant number BRA2016119, Q Zhang) and the Changzhou Sci \& Tech Program (grant number CJ20179031, Q Zhang). We thank Beijing Medintell Bioinformatic Technology Co., Ltd for assistance in high-throughput sequencing and data analysis. The authors have no other relevant affiliations or financial involvement with any organization or entity with a financial interest in or financial conflict with the subject matter or materials discussed in the manuscript apart from those disclosed.

No writing assistance was utilized in the production of this manuscript.

\section{Ethical disclosure}

The authors state that they have obtained appropriate institutional review board approval or have followed the principles outlined in the Declaration of Helsinki for all human or animal experimental investigations. In addition, for investigations involving human subjects, informed consent has been obtained from the participants involved.

\section{Open access}

This work is licensed under the Attribution-NonCommercial-NoDerivatives 4.0 Unported License. To view a copy of this license, visit http://creativecommons.org/licenses/by-nc-nd/4.0/ 
Summary points

- Compared with normal control, 962 differentially expressed mRNAs (DEmRNAs), 545 differentially expressed long noncoding RNAs (DElncRNAs) and 53 differentially expressed miRNAs (DEmiRNAs) were identified in asthma; 1328 DEmRNAs, 608 DEIncRNAs and 63 DEmiRNAs were identified in severe asthma.

- A total of 95 DEmRNAs, 143 DEIncRNAs and 96 DEmiRNAs were identified between asthma and severe asthma.

- Fc $\gamma$ R-mediated phagocytosis and spliceosome were significantly enriched pathways for DEmRNAs derived from shared and severe asthma-specific DEmiRNA-DEmRNA-DEIncRNA interaction network, respectively.

- MiR-133a-3p-EFHD2/CNN2-AC144831.1 interactions might play an important role in both asthma and severe asthma.

- MiR-3613-3p-CD44/BCL11B-LINC00158/CTA-217C2.1/AC010976.2/RP11-641A6.2 interactions and miR-93-5pBCL11B-LINC00158/CTA-217C2.1/AC010976.2/RP11-641A6.2 interactions were speculated to involve with the development of asthma to severe asthma.

\section{References}

Papers of special note have been highlighted as: $\bullet$ of interest; $\bullet \bullet$ of considerable interest

1. Masoli M, Fabian D, Holt S, Beasley R. The global burden of asthma: executive summary of the GINA Dissemination Committee report. Allergy 59(5), 469-478 (2004).

2. Hufnagl K, Hirt R, Robibaro B. Out of breath: asthma in humans and their animals. Comparative Med. 71-85 (2017).

3. Chung KF, Wenzel SE, Brozek JL et al. International ERS/ATS guidelines on definition, evaluation and treatment of severe asthma. Eur. Respir. J. 43(2), 343-373 (2014).

- Indicates that definition, evaluation and treatment of severe asthma.

4. Hekking PP, Wener RR, Amelink M, Zwinderman AH, Bouvy ML, Bel EH. The prevalence of severe refractory asthma. J. Allergy Clin. Immunol. 135(4), 896-902 (2015).

5. Antonicelli L, Bucca C, Neri M et al. Asthma severity and medical resource utilisation. Eur. Respir. J. 23(5), 723-729 (2004).

6. Chipps B, Kianifard F, Viduarre CF, Massanari M. Effect of omalizumab on peripheral blood eosinophils in children with moderate-severe persistent allergic asthma [Abstract]. Chest 136(4), 35S (2009).

7. Proceedings of the ATS workshop on refractory asthma: current understanding, recommendations, and unanswered questions. American Thoracic Society. Am. J. Respir. Crit. Care Med. 162(6), 2341-2351 (2000).

8. Hambly N, Nair P. Monoclonal antibodies for the treatment of refractory asthma. Curr. Opin. Pulm. Med. 20(1), 87-94 (2014).

9. Sadatsafavi M, Lynd L, Marra C et al. Direct health care costs associated with asthma in British Columbia. Can. Respir. J. 17(2), 74-80 (2010).

10. Hwang HW, Mendell JT. MicroRNAs in cell proliferation, cell death, and tumorigenesis. Br. J. Cancer 96(Suppl), R40-44 (2007).

11. Cao J. The functional role of long non-coding RNAs and epigenetics. Biol. Proced. Online 16, 11 (2014).

12. Chinese Medical Association Respiratory Diseases Association Asthma Group. Chinese bronchial asthma prevention and treatment guidelines (basic version). Zhonghua Jie He He Hi Xi Za Zhi 36(5), 331-336 (2013).

13. Chinese Medical Association Respiratory Diseases Association Asthma Group. Expert consensus on diagnosis and treatment of refractory asthma. Zhonghua Jie He He Hi Xi Za Zhi 33(8), 1-7 (2010).

14. Bonasio R, Shiekhattar R. Regulation of transcription by long noncoding RNAs. Annu. Rev. Genet. 48, 433-455 (2014).

15. Moran VA, Perera RJ, Khalil AM. Emerging functional and mechanistic paradigms of mammalian long non-coding RNAs. Nucleic Acids Res. 40(14), 6391-6400 (2012).

16. Chiba Y, Tanoue G, Suto R et al. Interleukin-17A directly acts on bronchial smooth muscle cells and augments the contractility. Pharmacol Rep. 69(3), 377-385 (2017).

-. Indicates that IL-17A upregulates RhoA protein through downregulation of miR-133a-3p, which is one of the causes of asthmatics.

17. Nigo YI, Yamashita M, Hirahara $\mathrm{K}$ et al. Regulation of allergic airway inflammation through toll-like receptor 4-mediated modification of mast cell function. Proc. Natl Acad. Sci. USA 103(7), 2286-2291 (2006).

18. Liang J, Jiang D, Griffith J et al. CD44 is a negative regulator of acute pulmonary inflammation and lipopolysaccharide-TLR signaling in mouse macrophages. J. Immunol. 178(4), 2469-2475 (2007).

19. Zhu J, Yamane H, Paul WE. Differentiation of effector CD4 T cell populations $\left(^{*}\right)$. Annu. Rev. Immunol. 28, 445-489 (2010).

20. Park SJ, Lee KS, Kim SR et al. Phosphoinositide 3-kinase delta inhibitor suppresses interleukin-17 expression in a murine asthma model. Eur. Respir. J. 36(6), 1448-1459 (2010). 
21. Schnyder-Candrian S, Togbe D, Couillin I et al. Interleukin-17 is a negative regulator of established allergic asthma. J. Exp. Med. 203(12), 2715-2725 (2006).

22. Jin R, Guo S, Wang MY et al. Administration of mycobacterial Ag85A and IL-17A fusion protein attenuates airway inflammation in a murine model of asthma. Int. Immunopharmacol. 17(4), 1067-1074 (2013).

23. Tian BP, Hua W, Xia LX et al. Exogenous interleukin-17A inhibits eosinophil differentiation and alleviates allergic airway inflammation. Am. J. Respir. Cell. Mol Biol. 52(4), 459-470 (2015).

24. Busse WW, Holgate S, Kerwin E et al. Randomized, double-blind, placebo-controlled study of brodalumab, a human anti-IL-17 receptor monoclonal antibody, in moderate to severe asthma. Am. J. Respir. Crit. Care Med. 188(11), 1294-1302 (2013).

25. Liang J, Jiang D, Jung Y et al. Role of hyaluronan and hyaluronan-binding proteins in human asthma. J. Allergy Clin. Immunol. 128(2), 403-411.e403 (2011).

26. Schroder NW, Maurer M. The role of innate immunity in asthma: leads and lessons from mouse models. Allergy 62(6), 579-590 (2007).

27. Lorentsen KJ, Cho JJ, Luo X et al. Bcl11b is essential for licensing Th2 differentiation during helminth infection and allergic asthma. Nat. commun. 9(1), 1679 (2018).

28. Karner J, Wawrzyniak M, Tankov $S$ et al. Increased microRNA-323-3p in IL-22/IL-17-producing T cells and asthma: a role in the regulation of the TGF-beta pathway and IL-22 production. Allergy 72(1), 55-65 (2017).

- Emphasizes that miR-323-3p acts in a negative feedback loop to control the production of IL-22 in IL-22/IL-17-producing T cells and might thus impact the T-cell responses in asthma. 
